EPJ Web of Conferences 59, 19004 (2013)

DOI: $10.1051 /$ epjconf/20135919004

(C) Owned by the authors, published by EDP Sciences, 2013

\title{
Frequency upshift via flash ionization phenomena using semiconductor plasma
}

\author{
A. Nishida ${ }^{1,2}$, M. Nakata ${ }^{3}$, T. Oba ${ }^{3}$, T. Higashiguchi ${ }^{2,3, a}$, N. Yugami ${ }^{2,3, b}$ \\ Y. Sentoku ${ }^{4, c}$ and R. Kodama ${ }^{1,2}$ \\ ${ }^{1}$ Graduate School of Engineering, Osaka University, 2-6 Yamada-oka, Suita, \\ Osaka 565-0871, Japan \\ 2 Japan Science and Technology Agency, CREST, 4-1-8 Honcho, Kawaguchi, \\ Saitama 332-0012, Japan \\ ${ }^{3}$ Department of Advanced Interdisciplinary Sciences, and Center for Optical Research \& \\ Education (CORE) Utsunomiya University, Yoto 7-1-2, Utsunomiya, Tochigi 321-8585, Japan \\ ${ }^{4}$ Department of Physics, University of Nevada, Mail Stop 220, Reno, Nevada 89506, USA
}

\begin{abstract}
We have demonstrated frequency upshift in the terahertz region by flash ionization. The magnitude of upshift frequency is tuned by the laser intensity. A proof of principle experiment has been performed with a plasma creation time scale much shorter than the period of the electromagnetic wave and a plasma length longer than its wavelength. Frequency upshifted from 0.35 to $3.5 \mathrm{THz}$ by irradiating a $\mathrm{ZnSe}$ crystal with a ultra-short laser pulse has been observed.
\end{abstract}

\section{INTRODUCTION}

Over-dense plasma can be easily created by ultra-short intense laser pulse. Electromagnetic (em) wave generated from such plasmas has a broad range of frequency which includes terahertz (THz) whose frequency is between traditional photonics and microwave. The $\mathrm{THz}$ region has many applications i.e, imaging, security, information-communication [1,2]. Most recent advances have been achieved by employing free electron lasers (FELs) to generate $\mathrm{THz}$ radiation [3]. FELs can generate pulse energies of the order of $\mu \mathrm{J}$ and operate over a wide frequency range. However, since they are large-scale systems they are not suitable for many applications.

A novel technique for tuning the frequency is flash ionization, which was predicted by S. C. Wilks et al $[4,5]$. The source em wave with an angular frequency $\omega_{0}$ is propagating in the $z$ direction. Plasmas are created in a much shorter time than the period of the source em wave along the propagation direction. The wave number of this source wave $k_{0}$ is fixed at the initial value. The angular frequency of the transmitted em wave adjusts to satisfy the dispersion relation in the plasma. The upshifted frequency $\omega_{f}$ is given by

$$
\omega_{f}^{2}=k_{0}^{2} c^{2}+\omega_{f}^{2}=\omega_{0}^{2}+\omega_{P}^{2}
$$

\footnotetext{
ae-mail: higashi@cc.utsunomiya-u.ac.jp

be-mail: yugami@cc.utsunomiya-u.ac.jp

ce-mail: sentoku@unr.edu
}

This is an Open Access article distributed under the terms of the Creative Commons Attribution License 2.0, which permits unrestricted use, distribution, and reproduction in any medium, provided the original work is properly cited. 


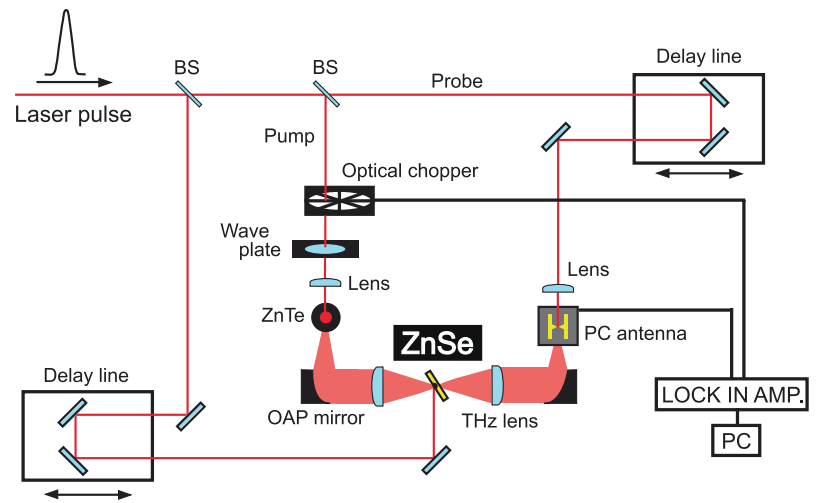

Figure 1. Experimental setup.

where $c$ is the speed of light and $\omega_{P}$ is the plasma frequency. Therefore, the magnitude of frequency upshift $\triangle \omega$ is expressed by

$$
\triangle \omega=\sqrt{\omega_{0}^{2}+\omega_{P}^{2}}-\omega_{0}^{2} .
$$

Thus, the output frequency can be tuned by adjusting the plasma density.

Following the publication of these theoretical predictions, several experiments were performed using microwaves and optical waves as sources [6-8]. However, these experiments do not fulfil the theory requirements. One is that the plasma creation time should be much shorter than the oscillation period of the em wave. The other is the plasma length is much longer than a wavelength to neglect the edge effect. In this paper, we have demonstrated the proof of principle experiment of flash ionization, which meets the requirements of the above two conditions.

\section{EXPERIMENTAL SETUP}

The experimental setup is shown in Fig. 1. The Ti:sapphire chirped-pulse amplification laser system delivered an maximum energy of $1 \mathrm{~mJ}$ at a center wavelength of $800 \mathrm{~nm}$, and $1 \mathrm{kHz}$ repetition rate. The laser beam was split into three beams. One beam is a pumping pulse to create electron carrier in a $\mathrm{ZnSe}$ crystal. The second beam is the chopped pump beam that was passed through the half wave plate and focused on a ZnTe crystal to generate the source THz wave. The third beam is used to detect the $\mathrm{THz}$ wave using a bow-tie photoconductive (PC) antenna. The source THz pulse is focused in the $\mathrm{ZnSe}$ crystal by the off-axis parabola (OAP) mirror and lens pair whose effective focal length is $76.2 \mathrm{~mm}$ and $150 \mathrm{~mm}$, respectively. The output THz pulse is collected by another OAP and lens pair, and finally focused on the PC antenna to detect the $\mathrm{THz}$ signal. We measured this small current by chopping the $0.5 \mathrm{kHz}$ optical pulse train that triggers the emitter and using a lock-in-amplifier.

A dashed red line in Fig. 2 indicates the waveform spectrum of the source THz wave. The central frequency of source wave is $0.35 \mathrm{THz}$. The laser pulse duration is $100 \mathrm{fs}$ that is much shorter than the source $\mathrm{THz}$ wave. Furthermore, the source $\mathrm{THz}$ wave has a wavelength of $0.85 \mathrm{~mm}$, which is shorter than the laser focal diameter $(10 \mathrm{~mm})$. Thus, the two theoretical requirements are perfectly satisfied in the present experiment.

\section{EXPERIMENTAL RESULTS AND DISCUSSION}

The solid line in Fig. 2 shows a typical upshifted spectrum. The upshifted spectrum is observed at $3.4 \mathrm{THz}$ with a frequency width of $0.3 \mathrm{THz}$. 


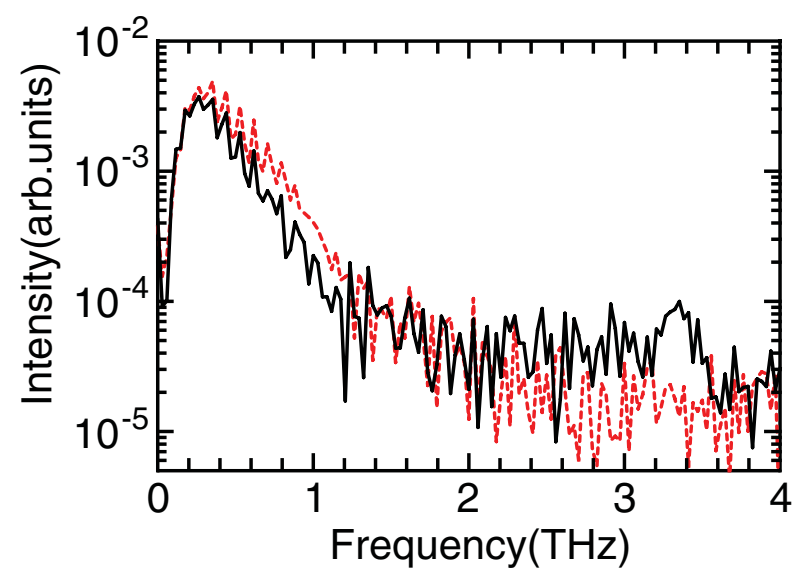

Figure 2. Measured upshifted frequency of THz pulses.

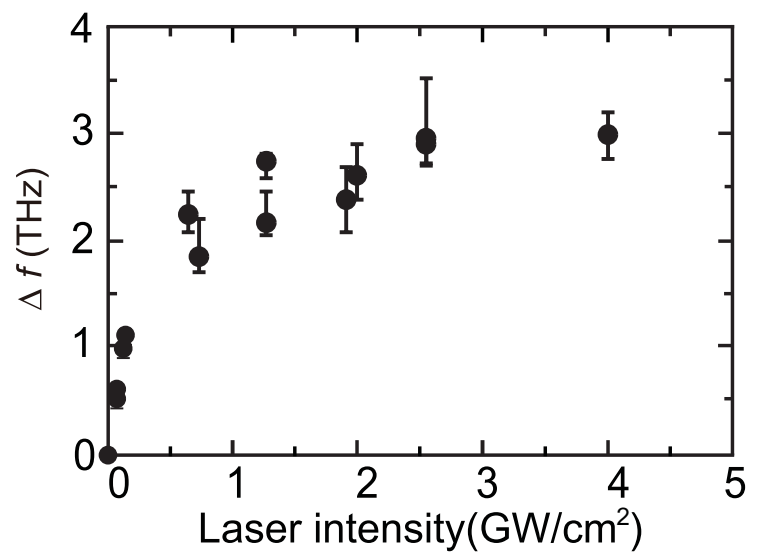

Figure 3. Observed frequency upshift as a function of the laser intensity.

Figure 3 shows the magnitude of upshift frequency $\triangle f$ changing by the pump laser intensity irradiated on the ZnSe crystal. The upshifted spectrum is observed up to $3.5 \mathrm{THz}$, when the laser intensity is $4.2 \mathrm{GW} / \mathrm{cm}^{2}$. The maximum frequency upshifted about 10 times as large as the seed $\mathrm{THz}$ pulse. The upper frequency limit of the measurement is due to the frequency response of the bow-tie type PC antenna as the detector.

Reference 5 indicates the relationship of energy conversion of the THz pulse. The maximum electric field of the upshifted wave is represented by

$$
E_{f \pm}=\frac{E_{0}}{2}\left(1 \pm \frac{\omega_{0}}{\omega_{f}}\right)
$$

The plus and minus signs denote copropagating and counterpropagating em waves, respectively. A proportional relationship between electric field and frequency is given by

$$
\frac{I_{f}}{I_{0}} \propto\left(\frac{E_{f}}{E_{0}}\right)^{2} \propto\left(\frac{\omega_{0}}{\omega_{f}}\right)^{2},
$$

The intensity of $\mathrm{THz}$ pulse $I$ is proportional to the square of the electric field strength $E$ which is detected by the PC antenna. The normalized ratio of the intensity of the THz pulse $I_{f} / I_{0}$ is plotted as 


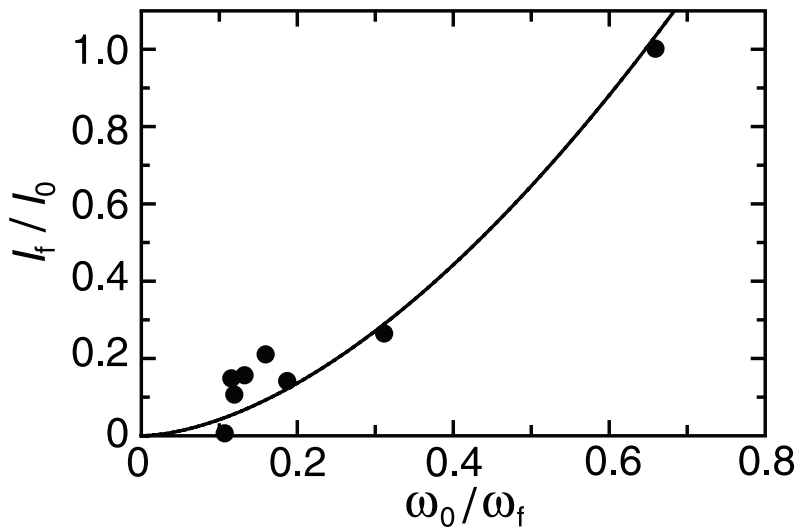

Figure 4. Nomarized intensity of THz pulse against angular frequency.

a function of the normalized frequency $\omega_{0} / \omega_{f}$. The fitting curve in Fig. 4 indicates $\propto\left(\omega_{0} / \omega_{f}\right)^{1.7}$. This experimental data is lower than the values given by Eq. (4) because the source THz pulse interacts not only with the surface of the ZnSe carrier plasma but also its interior.

\section{CONCLUSION}

In conclusion, we have demonstrated the proof of principle experiment of the flash ionization with the $\mathrm{ZnSe}$ crystal. The plasma creation time is much shorter than the period of the source EM wave and plasma size is much larger than a cycle of $\mathrm{THz}$ wave. We upshifted the frequency from 0.35 to $3.5 \mathrm{THz}$. Various frequencies can be observed by changing pumping laser intensity irradiated on the ZnSe crystal. We confirmed relationship between the electric field strength of upshifted THz pulse and its intensity. This result has possibilities of tunable high power radiation source in the $\mathrm{THz}$ region.

The authors are grateful to Takamitsu Otsuka, Hiroaki Kashiwazaki, and Jun Miyazawa for their unparalleled technical support. This work was supported by Core Research for Evolutional Science and Technology (CREST) of the Japan Science and Technology Agency (JST). It was also supported by the Japan Society for the Promotion of Science Global Centers of Excellence program (GCOE). A part of this work was performed under the auspices of MEXT (Ministry of Education, Culture, Science and Technology, Japan). We also are grateful to the Cooperative Research Center and the Venture Business Laboratory (VBL) of Utsunomiya University for providing the laser system.

\section{References}

[1] B. Ferguson, X.-C. Zhang, Nature Mat. 1, 26 (2002)

[2] M. Tonouchi, Nature Photo. 1, 97 (2007)

[3] G. M. H. Knippels, X. Yan, A. M. MacLeod, et al., Phys. Rev. Lett. 83, 1578 (1999)

[4] C. Lai, T. Katsouleas, W. Mori, et al., Plasma Science, IEEE Transactions. 21, 45 (1993)

[5] S. Wilks, J. Dawson, W. Mori, Phys. Rev. Lett. 61, 337 (1988)

[6] S. Kuo, Phys. Rev. Lett. 65, 1000 (1990)

[7] C. Joshi, C. Clayton, K. Marsh, et al., Plasma Science, IEEE Transactions. 18, 814 (1990)

[8] N. Yugami, T. Niiyama, T. Higashiguchi, et al., Phys. Rev. E 65, 1(2002) 